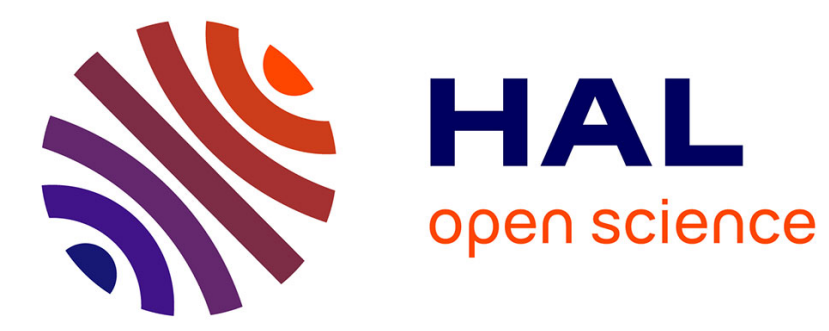

\title{
State and parameter estimation for a class of schistosomiasis models
}

Derdei Bichara, Aboudramane Guiro, Abderrahman Iggidr, Diène Ngom

\section{To cite this version:}

Derdei Bichara, Aboudramane Guiro, Abderrahman Iggidr, Diène Ngom. State and parameter estimation for a class of schistosomiasis models. Mathematical Biosciences, 2019, 315, 10.1016/j.mbs.2019.108226 . hal-02189643

\section{HAL Id: hal-02189643 \\ https://hal.inria.fr/hal-02189643}

Submitted on 19 Jul 2019

HAL is a multi-disciplinary open access archive for the deposit and dissemination of scientific research documents, whether they are published or not. The documents may come from teaching and research institutions in France or abroad, or from public or private research centers.
L'archive ouverte pluridisciplinaire HAL, est destinée au dépôt et à la diffusion de documents scientifiques de niveau recherche, publiés ou non, émanant des établissements d'enseignement et de recherche français ou étrangers, des laboratoires publics ou privés. 


\title{
State and Parameter Estimation for a Class of Schistosomiasis Models
}

\author{
Derdei M. Bichara ${ }^{1}$, Aboudramane Guiro ${ }^{2}$, Abderrahman Iggidr $^{3}$, Diene Ngom $^{4}$
}

${ }^{1}$ Department of Mathematics, California State University, Fullerton, CA 92831, USA ${ }^{2}$ Laboratoire d'Analyse et d'Informatique (LAMI), Département de Mathématiques UFR/ST, Université Nazi Boni, Bobo-Dioulasso, Burkina Faso

${ }^{3}$ Université de Lorraine, CNRS, Inria, IECL, F-57000 Metz, France

${ }^{4}$ Département de Mathématiques, Université Assane Seck de Ziguinchor BP 523 Ziguinchor, Sénégal. UMI 2019-IRD \& UMMISCO-UGB

\begin{abstract}
We develop a general framework to estimate the proportion of infected snails and snail-human transmission parameter of a class of models that describes the evolution of schistosomiasis. To do so, we consider simultaneously the dynamics of schistosomiasis, captured by the homogeneous version of the classical MacDonald's model, and the measurable output: the number of female schistosomes per single host. The proposed method consists of designing an auxiliary dynamical system, called observer, whose solutions converge exponentially to those of the system capturing the schistosomiasis model. Moreover, we derive an estimation of the snail-human transmission rate, an unknown but key parameter in the dynamics of schistosomiasis. These estimations are central in two of the strategies of controlling schistosomiasis, namely the use of molluscicides and mass drug administration. To further investigate control strategies on a larger scale, we consider a heterogeneous model which consists of an arbitrary number of human groups or patches and an arbitrary number of fresh-water sources, natural habitats of snails. Provided that the data of infected humans' worm burden in each patch or group is available, we provide a method of estimating the proportion of infected snails in each snail natural habitat, thereby providing a map on where to implement control strategy to mitigate or eliminate Schistosomiasis.
\end{abstract}

Mathematics Subject Classification: 92D25, 92D30, 93B05, 93B07, 93C40.

Keywords: Schistosomiasis, MacDonald's model, observer, state estimation, parameter estimation, control strategies. 


\section{Introduction}

Schistosomiasis, also known as bilharzia, is a major parasitic disease that causes over 200 million in morbidity, and over 700 million people are at risk across the world [18, 54]. The disease is prevalent mostly in tropical and subtropical areas and falls under the umbrella of neglected tropical diseases and is topped only by malaria in terms of incidence and economic burden in endemic regions. Consequently, understanding the mechanisms that drive the transmission Schistosomiasis and providing tools to control it should be a worldwide priority.

Schistosomiasis is the end-product of a complex biological cycle that involves two hosts - humans and snails; two free-living transmission pathogen, namely, cercariae and miracidia; and a variety of environments. Indeed, the overall transmission goes as follows: Schistosoma eggs are excreted from humans into the environment via urine or faeces (depending on the species of schistosomes). The eggs hatch into miracidia after a contact with fresh water. These miracidia infect water snails by penetrating the latter through their soft tissue, which results in amplification of the numbers of parasites by asexual reproduction. The parasite leaves the snail intermediate host as cercariae which can penetrate the skin of humans in contact with water, thus completing the life cycle.

Different intervention strategies have been implemented in an attempt to eradicate schistosomiasis or alleviate its morbidity in many endemic countries [34]. Indeed, mass drug administration campaign was widely used with varying degrees of success. However, this method has its own set of challenges due factors such as the development of drug resistance [34], the difficulties of sustaining coverage of a critical mass of the target population, and the scarcity of resources to undertake nationwide programs $[26,47]$. The second control strategy consists of controlling the intermediate host snails using chemical molluscicides. However, there are two main challenges to this control strategy: assessing the number of infected snails in a given fresh-water source, and assessing which snail habitats to target. The goal of this paper is to address these two issues.

The use of mathematical models in understanding the dynamics of the interactions between host and vectors goes back to Ross' 1911 paper [41]. Indeed, in [41], Ross provides a model capturing the overall dynamics of the malaria, a disease caused by the parasite Plasmodium falciparum and transmitted between human hosts and female Anopheles mosquitoes when the latter bites the former. As mentioned above, schistosomiasis has a somehow similar but more complex transmission cycle and its mathematical model was first derived by MacDonald [37] and later developed in different directions (see $[4,8,9,40,51,52]$ and the references therein). However, estimating the state variables and/or parameters of the model is a perennial problem in epidemiology in general and in understanding the dynamics of schistosomiasis in particular. For instance, some parameters in schistosomiasis models, such as the life span of snails, could be obtained experimentally (2-3 years) [24, 48, 51] or the life span of the human hosts. Some other parameters, however, are unknown and very difficult to estimate. This

the case for the snail-human transmission rate. Indeed, this parameter represents a simplified com- 
pound value of a complex biological cascade of events leading to the infection [9]. And so, estimating the value of this snail-human transmission term is crucial in a control strategy that consists of blocking the transmission of schistosomiasis. Furthermore, a facet of this strategy is the chemical control strategy, in which chemical molluscicides are used to target infected snails in a given environment. This is also a difficult task as it requires one to distinguish between uninfected and infected snails in natural environments. Although some ad hoc estimations of the snail-human transmission rate exist for particular cases [24, 53], a comprehensive and general method of estimating the snail-human transmission is lacking. Moreover, to the best of our knowledge, there is no method in the literature that estimates the infected snail in water source.

In this paper, we use elements of control theory to estimate the proportion of infected snails in a given freshwater source and the snail-human transmission rate. To this end, we consider the dynamics of schistosomiasis along with the measurable output. For schistosomiasis' dynamical model, we consider the classical MacDonald's model [8, 9, 37] and we assume that the measurable output is the state variable that represents the number of female schistosomes. Indeed, for schistosomiasis, it is widely reported that it is possible to measure the number of parasites per hosts $[8,13,51,52]$, using faeces or urine.

Therefore, the first focal point of this paper is to develop a general framework to estimate the proportion of infected snails and the snail-human transmission parameter using the available data and the temporal evolution of schistosomiasis. More precisely, the method consists of constructing an observer. An observer is essentially an auxiliary dynamical system that uses the available data on some state variables and/or parameters and delivers dynamical estimates of the unmeasured state variables. The solutions of this auxiliary dynamical system must converge (as fast as possible) towards the solutions of the original system. The notion of observers originates in the theory of systems [36]. The use of this powerful tool of control theory is recent in epidemiology [1, 2, 3, 7, 14, 15, 16, 17, 22, 23, 33, 44, 45, 49]. In estimating the unmeasured proportion of infected snails and the unknown snail-human transmission rate, our framework allows a better understanding of the schistosomiasis dynamics and better implementation of snail control strategies. After the preliminaries on the observers (Section 2), the bulk of this part is described in Section 3.

The second focal point of the paper is to address schistosomiasis transmission on a larger scale. Indeed, schistosomiasis, also known as a disease of poverty, is endemic in developing countries where resources are very limited and snail habitats (ponds, marshland, fresh-water sources, ...) are widespread. And so, determining which snail habitats to target for biological control or which human age-group(s) or patch(es) to target for mass drug administration, is a major problem. To deal with this question, we consider a heterogeneous schistosomiasis model, derived originally by [40] (see also [9, 38]). The model consists of human hosts, structured into $l$ groups or patches, interacting with snails, structured into $m$ natural habitats (ponds, fresh-water, etc). Assuming that we could measure the worm burden in hosts in the $l$ host group - this is a routine technique available in most countries - our method provides 
a tool to estimate the proportion of infected snails in each snail habitat, thereby providing a map to optimize resource allocation in controlling schistosomiasis. This part is treated in Section 4.

\section{Preliminaries}

In this section we briefly introduce the notion of observer. Let us consider a phenomenon whose evolution is captured by a dynamical system:

$$
\dot{x}=f(x, \theta)
$$

where $x$ is the vector representing state variables and $\theta$ the vector of parameters of the considered model. Suppose that only some or a combination of state variables are available as measurements. In control theory nomenclature, this quantity is called measurable output and is denoted by $y=h(x)$ where $h$ is a smooth function of the state $x$.

Hence the input-output system that accounts for the dynamics of the system along with the available data is given by:

$$
\left\{\begin{array}{l}
\dot{x}=f(x, \theta), \\
y=h(x) .
\end{array}\right.
$$

An observer is an auxiliary dynamical system that takes into account the available output into the dynamics of the system and that converges asymptotically to the original system (1). Particularly, if the vector of parameters $\theta$ is known, an observer of System (1) has the following form:

$$
\left\{\begin{array}{l}
\dot{\hat{z}}(t)=g(\hat{z}(t), y(t), \theta) \\
\hat{x}=k(\hat{z}(t), y(t), \theta)
\end{array}\right.
$$

The challenge in designing an observer consists of finding the two functions $g$ and $k$ in such a way that the solutions of System (3) converge exponentially to that of System (2), irrespective of initial conditions. That is, the functions $g$ and $k$ must satisfy, for any initial condition $\hat{x}\left(t_{0}\right)$ :

$$
\|x(t)-\hat{x}(t)\| \leq e^{-\lambda t}\left\|x\left(t_{0}\right)-\hat{x}\left(t_{0}\right)\right\|
$$

for some $\lambda \in \mathbb{R}_{+}$. When System (1) is linear, the problem of finding an observer is completely solved by the Luenberger observer [36], and the possibility of achieving arbitrary fast estimation is equivalent to the so-called observability property (see below Definition 2.3 and Theorem 2.1). If the considered system is non-linear, typically, there is no "off the shelf" method to design an observer (see [11] and the references therein for an overview on the observer theory, and [32] for a short survey of observability and observers with applications to various life-support-systems).

Throughout the rest of the paper, we will use some terminologies and notions that we recall in the rest of section.

When the vector of parameters $\theta$ is not fully known, it may still be possible to design an observer for System (2). These type of observers are defined as follows: 
Definition 2.1. An adaptive observer for System (2) is an observer for joint estimation of the state $x(t)$ and the parameter $\theta$.

An (exponential) adaptive observer for System (2) is a dynamical system of the form

$$
\left\{\begin{array}{l}
\dot{\hat{z}}(t)=g_{1}(\hat{z}(t), \hat{\theta}(t), y(t)) \\
\dot{\hat{\theta}}(t)=g_{2}(\hat{z}(t), \hat{\theta}(t), y(t)) \\
\hat{x}(t)=k(\hat{z}(t), \hat{\theta}(t), y(t))
\end{array}\right.
$$

such that the solutions of (4) and those of (2) satisfy $\hat{x}(t)-x(t)$ and $\hat{\theta}(t)-\theta$ converge (exponentially fast) to zero as time $t$ goes to infinity.

The following two definitions and theorem will be used to prove some of the results in Section 3 and Section 4.

Definition 2.2. A square matrix $A$ is said to be Hurwitz (or stable) if all its eigenvalues have negative real parts.

Definition 2.3 (Kalman observability rank condition [50]). Let $A$ be a $n \times n$ matrix and $C$ be a $q \times n$ matrix. The pair $(A, C)$ is said to be observable or to satisfy the Kalman observability rank condition if the matrix:

$$
\mathcal{O}_{(C, A)}=\left(\begin{array}{c}
C \\
C A \\
C A^{2} \\
\vdots \\
C A^{n-1}
\end{array}\right)
$$

is of rank $n$.

Theorem 2.1 (Pole-Shifting Theorem [50, page 61]). If the pair $(A, C)$ satisfies the Kalman observability rank condition then it is possible to find a matrix $K$ in such a way that the eigenvalues of the matrix $A-K C$ are all with negative real part.

For linear systems of the form:

$$
\left\{\begin{array}{l}
\dot{x}(t)=A x(t) \\
y(t)=C x(t),
\end{array}\right.
$$

Definition 2.3 provides a characterization of the observability of the corresponding linear system (5). That is, given the measurable output $y$, it is possible to determine the behavior of the entire system $x$ from the system's output, for all $t \in[0, T]$. Theorem 2.1 guarantees the existence of a matrix $K$ such that the spectrum of $A-K C$ belongs to the open left half-plane of $\mathbb{C}$, for an observable pair $(A, C)$. Therefore, if the linear system (5) satisfies the Kalman observability rank condition then an exponential observer is simply given by:

$$
\dot{\hat{x}}(t)=A x(t)-K(C \hat{x}(t)-y(t))
$$


since the error $e(t)=\hat{x}(t)-x(t)$ satisfies $\dot{e}(t)=(A-K C) e(t)$ and the matrix $K$ can be chosen in such a way that the matrix $A-K C$ is Hurwitz.

In the following section, we design an observer for a schistosomiasis model in order to estimate the number of infected snails and transmission rate from snails to human hosts.

\section{Observer design for a schistosomiasis model}

Let $w$ be the number of female schistosomes (worms) per single host and $s$ the proportion of infected snails. The female schistosomes per single host decays at a per capita rate $\gamma$ and are replenished at a rate $a$. The latter process is proportional to the proportion of infected snails $s$. The proportion of susceptible snails $1-s$ could be infected through an indirect contact with schistosomes that are excreted from hosts $w$, and naturally die at a rate $\mu$. Finally, the overall dynamics of the schistosomiasis is captured by the following model:

$$
\left\{\begin{array}{l}
\dot{w}=-\gamma w+a s \\
\dot{s}=b(1-s) w-\mu s
\end{array}\right.
$$

Model (6) is a version of MacDonald's model and is proposed first by Barbour [9] in investigating the dynamics of bilharzia - See also $[8,9]$ for a more detailed formulation of the model, and $[4,10]$ for a literature review. It is easy to show that the compact set

$$
\Omega=\left\{(w, s) \in \mathbb{R}^{2} \mid 0 \leq w \leq \frac{a}{\gamma} ; 0 \leq s \leq 1\right\}
$$

is a positively invariant and absorbing set for System (6), making the solutions biologically substantiated.

It has been documented that the number of female schistosomes (worms) per single host could be measured using urine or faeces samples [8, 13]. Particularly, authors in [13] provided data of the numbers of schistosome eggs per gram of feces per host (Fig 1 in [13]). This is a widespread diagnosis technique for parasitological infections in general and schistosomiasis in particular. We suppose henceforth that the state variable $w$ in Model (6) is measured. Thus, the measurable output is $y(t)=w$. By denoting $x=(w, s)^{T}$, the dynamical system that describes the evolution of schistosomiasis along with the available data could be written in a compact form as follows:

$$
\left\{\begin{array}{l}
\dot{x}=M x+U(y) x \\
y=C x
\end{array}\right.
$$

where $M=\left(\begin{array}{cc}-\gamma & a \\ b & -\mu\end{array}\right), U(y)=\left(\begin{array}{cc}0 & 0 \\ 0 & -b y\end{array}\right)$, and $C=(1,0)$. As many models representing a complex system, some of the parameters of Model (8) are unknown. For instance, the death rates of schistosomes $\gamma$, and infected snails $\mu$, respectively, could be measured by biological considerations (see [19]). However, transmission parameters are usually unknown, and the existing proposed values are ad hoc. 
In the next two subsections, we design observer to estimate the state variable $s$ in two cases: when all the parameters are known, and when only one of the transmission parameters is known. Actually, the proposed observers provide tools to estimate the state variable $s$ without using the above-mentioned transmission parameters $a$. Moreover, we use this observer to estimate this parameter.

\subsection{State variable estimate when all parameters are known}

The following theorem provides an observer (state-estimator) using an output-injection for the system (8).

Theorem 3.1. An exponential estimator for the MacDonald's Bilharzia model (6) with measurable output $y(t)=w(t)$ is given by

$$
\dot{\hat{x}}=M \hat{x}+U(y) \hat{x}-K(C \hat{x}-y)
$$

where $K^{T}=\left(k_{1}, a+b\right)$ for any positive constant $k_{1}$.

Proof.

It suffices to show that the trajectories of System (9) converge exponentially to those of System (8) for any initial conditions of System (9). To do so, we show that the equation describing the evolution of the error $e(t)=\hat{x}-x$ converges exponentially to zero. Indeed,

$$
\begin{aligned}
\dot{e} & =\dot{\hat{x}}-\dot{x} \\
& =M \hat{x}+U(y) \hat{x}-K(C \hat{x}-y)-M x-U(y) x \\
& =(M-K C) e+U(y) e
\end{aligned}
$$

Let us consider the Lyapunov function $\mathcal{L}=\frac{1}{2} e^{T} e$. The derivative of $\mathcal{L}$ along the trajectories of (10) is:

$$
\begin{aligned}
\dot{\mathcal{L}} & =\frac{1}{2} \dot{e}^{T} e+\frac{1}{2} e^{T} \dot{e} \\
& =\frac{1}{2}[(M-K C) e+U(y) e]^{T} e+\frac{1}{2} e^{T}[(M-K C) e+U(y) e] \\
& =\frac{1}{2}\left[e^{T}(M-K C)^{T}+e^{T} U^{T}(y)\right] e+\frac{1}{2} e^{T}[(M-K C) e+U(y) e] \\
& =\frac{1}{2} e^{T}\left[(M-K C)^{T}+(M-K C)\right] e+\frac{1}{2} e^{T}\left[U^{T}(y)+U(y)\right] e
\end{aligned}
$$

Using the expressions of $M, K, C$ and $U$ given in (8), Equation (11) leads to:

$$
\begin{aligned}
\dot{\mathcal{L}}(e) & =e^{T}\left(\begin{array}{cc}
-k_{1}-\gamma & 0 \\
0 & -\mu-b y
\end{array}\right) e \\
& \leq 0
\end{aligned}
$$

since $y \geq 0$. Moreover, $\dot{\mathcal{L}}$ is a quadratic negative-definite function. It follows that, by Lyapunov's theorem all trajectories of (10) approach the origin with an exponential convergence rate as $t$ goes to infinity. We deduce therefore that System (9) is an exponential observer of System (8). 


\section{Remark 3.1.}

In Theorem 3.1 we chose $K^{T}=\left(k_{1}, k_{2}\right)$ with $k_{2}=a+b$. However $K$ could be any vector that makes $M-K C$ Hurwitz.

We carry out numerical simulations to showcase how the overall behavior of the solutions of the original system (8) compared to the observer (9). Unless otherwise stated, we use the following parameters: $\gamma=0.05$ day $^{-1}, \mu=0.04$ day $^{-1}, a=2$ female worm $\times$ day $^{-1}$ and $b=0.01$ female worm ${ }^{-1} \times$ day $^{-1}$. The initial conditions $(w(0), s(0))=(3,0.3)$ and $(\hat{w}(0), \hat{s}(0))=(1,0.1)$ are used for the systems (8) and (9), respectively. Figure 1 displays the dynamics of infected snails $s$ (red), given by Model (6), and its estimated value, $\hat{s}$ (blue), obtained using the observer (9). As anticipated, the curves of $\hat{s}$ and that of $s(t)$ merge rather quickly (convergence-time $\approx 7$ days). Moreover, the merger occurs way before the two systems reach the equilibrium value (dashed green). It is worthwhile to note that the trajectories given by the observer (9) converge to those of Model (6), for any initial conditions of the observer system. This avoids the often difficult task of estimating the initial conditions to integrate epidemic systems.

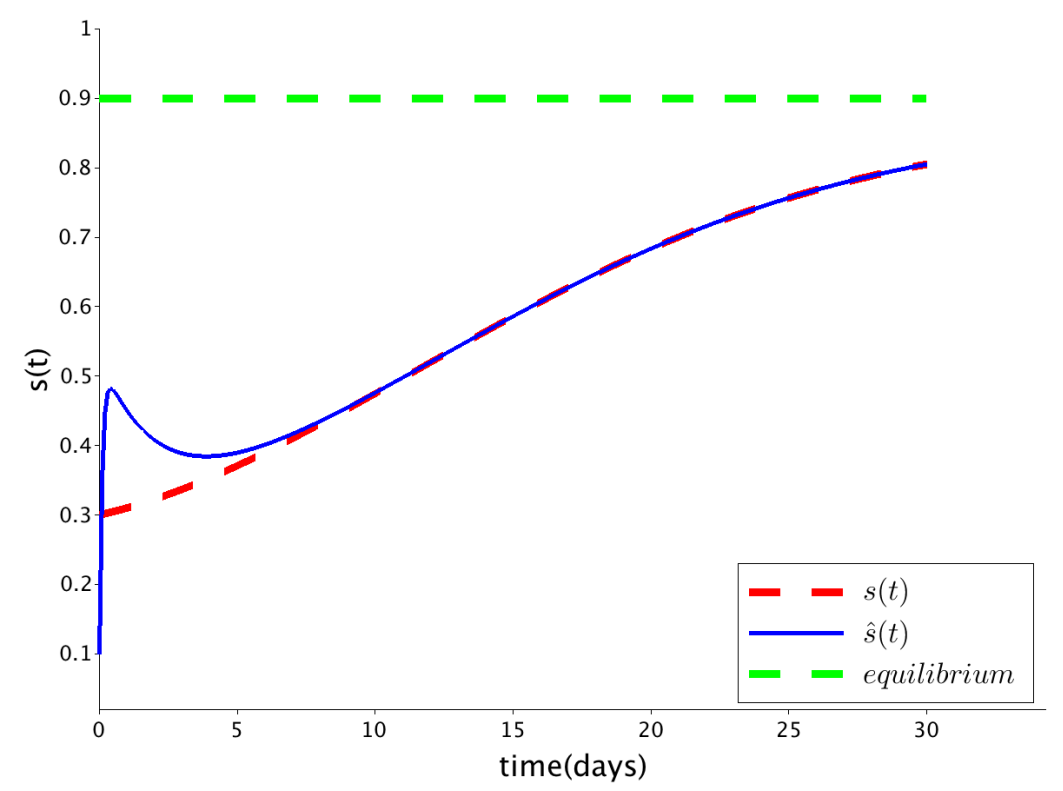

Figure 1: Coupled dynamics of the proportion of infected snails $s(t)$ (dashed red) as given by System (8) and its estimate $\hat{s}(t)$ (blue) as given by the observer (9).

\subsection{Adaptive observer: A tool to estimate both state variables and pa- rameters}

In Section 3.1, we designed an observer to estimate a state variable, namely the proportion of infected snails $s$, given that all the parameters of the model are known. Indeed, the observer (9) depends on 


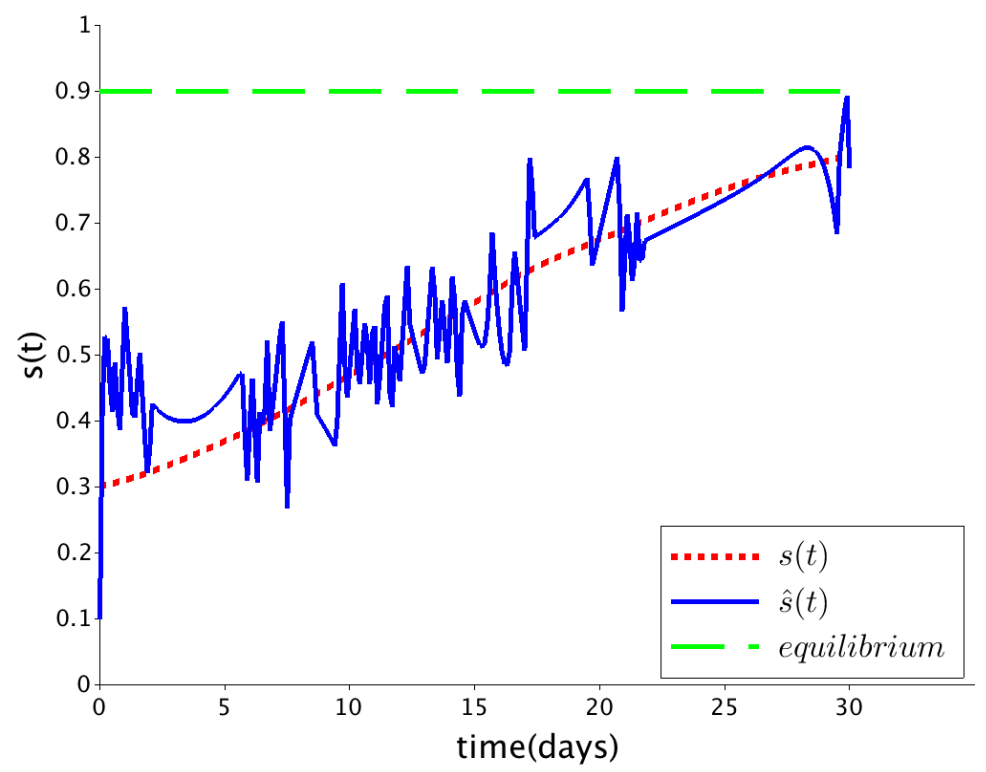

Figure 2: Coupled dynamics of the proportion of infected snails $s(t)$ (dotted red curve) as given by System (8) and its estimate $\hat{s}(t)$ (blue) as given by the observer (9) when the measurements y(t) are corrupted by noise.

all the parameters of Model (6). However, unfortunately the values of some parameters in epidemic models are usually not known. A perennial problem in epidemic models is the estimation of the transmission parameters. For the schistosomiasis model, it is the parameter $a$ which represents the snail-host infection rate that is difficult to estimate [9]. In this section, we design an observer that allows to estimate simultaneously the state variable $s$ and the snail-host transmission rate $a$ given that $w$ is measured. Such observer is called adaptive observer (see for instance [12] and [11], chap. 7). Let $a$, the unknown parameter. Using the linear transformation $\left(z_{1}, z_{2}\right)^{T}=(w,-\gamma w+a s)^{T}=T x$, where $T=\left(\begin{array}{cc}1 & 0 \\ -\gamma & a\end{array}\right)$, Model (6) can be written, with the new coordinates as

$$
\left\{\begin{array}{l}
\dot{z}(t)=(A+B(y)) z(t)+\psi(y(t)) a \\
y=C_{0} z
\end{array}\right.
$$

where $A=\left(\begin{array}{cc}0 & 1 \\ -\gamma \mu & -(\mu+\gamma)\end{array}\right), B(y)=-b y\left(\begin{array}{cc}0 & 0 \\ \gamma & 1\end{array}\right), \psi(y)=\left(\begin{array}{c}0 \\ b y\end{array}\right)$, and $C_{0}=(1,0)$. 
Let $\lambda$ be any positive real number. Define the following functions of the output $y:=y(t)$ :

$$
\left\{\begin{aligned}
k_{1}(y) & =3(\lambda+1)-\gamma-\mu-b y \\
k_{2}(y) & =\gamma^{2}+\mu^{2}+\gamma \mu+b^{2} y^{2}+(2 \mu+\gamma) b y+3 \lambda(\lambda+2)+2-3(\lambda+1)(b y+\mu+\gamma) \\
& =-3(\mu+\gamma)+\gamma^{2}+\mu^{2}+\gamma \mu+b^{2} y^{2}+(2 \mu+\gamma-3-3 \lambda) b y+3 \lambda(\lambda+2)+2-3 \lambda(\mu+\gamma) \\
\Lambda(y) & =\frac{\lambda(\lambda+1)(\lambda+2)}{b y}
\end{aligned}\right.
$$

The expression of $\Lambda(y)$ in (13) is well defined since $y(t)>0$ for all $t \geq 0$ if $y(0)>0$. Indeed, $y$ is the measurable output and satisfies the equation $\dot{y}=-\gamma y+a s$. Moreover, we assume that the ecosystem is in an endemic situation which means that $\mathcal{R}_{0}=\frac{a b}{\gamma \mu}>1$ and implies [9] that $y(t) \rightarrow \bar{y}=\frac{a}{\gamma}\left(1-\frac{\gamma \mu}{a b}\right)>0$. The following theorem gives an observer that estimates both the states variables and the transmission parameter $a$.

\section{Theorem 3.2.}

An exponential adaptive observer for System (12) is given by the following system:

$$
\left\{\begin{array}{l}
\dot{\hat{z}}(t)=(A+B(y)) \hat{z}(t)+\psi(y(t)) \hat{a}(t)-K(y(t))\left(C_{0} \hat{z}(t)-y(t)\right), \\
\dot{\hat{a}}(t)=-\Lambda(y(t))\left(C_{0} \hat{z}(t)-y(t)\right)
\end{array}\right.
$$

where $K(y(t))=\left(k_{1}(y(t)), k_{2}(y(t))\right)^{T}$ and $\Lambda(y(t))$ are defined by relations $(13)$.

The proof of Theorem 3.2 uses a classical result concerning a class of time-varying linear systems, which we recall here:

Theorem 3.3 ([20], Chap. IV, Theorem 2). Let D be a constant matrix whose characteristic roots $\lambda_{1}, \ldots, \lambda_{n}$ are all simple, and let $\xi_{i}$ be a characteristic vector of $D$ belonging to the characteristic root $\lambda_{i}(i=1, \ldots, n)$. If $R(t)$ is a continuous matrix defined for $t \geq t_{0}$ such that

$$
\int_{t_{0}}^{\infty}|R(t)| d t<\infty
$$

then the equation $\dot{x}=(D+R(t)) x$ has a fundamental system of solutions $x_{1}(t), x_{2}(t), \ldots, x_{n}(t)$ satisfying for $t \rightarrow \infty$

$$
x_{k}(t) \sim e^{\lambda_{k} t} \xi_{k}, \quad(k=1, \ldots, n) .
$$

The symbol $\sim$ in Theorem 3.3 denotes the asymptotic equivalence.

Proof of Theorem 3.2.

Recall that the goal is to show that the error between System (12) and System (14) converges exponentially to zero. The estimation error $e(t)=\left(e_{1}(t), e_{2}(t), e_{a}(t)\right)^{T}=\left(\hat{z}_{1}(t)-z_{1}(t), \hat{z}_{2}(t)-z_{2}(t), \hat{a}(t)-a(t)\right)^{T}$ 
satisfies

$$
\begin{aligned}
\dot{e}(t) & =\left(\begin{array}{ccc}
-k_{1}(y(t)) & 1 & 0 \\
-k_{2}(y(t))-\gamma \mu-b \gamma y(t) & -\gamma-\mu-b y(t) & b y(t) \\
-\Lambda(y(t)) & 0 & 0
\end{array}\right) e(t) \\
& :=M(y(t)) e(t) .
\end{aligned}
$$

The time-varying matrix $M(y)$ could be written as

$$
M(y)=\tilde{A}(y)-\tilde{K}(y) \tilde{C}
$$

with $\tilde{A}(y)=\left(\begin{array}{ccc}0 & 1 & 0 \\ -\gamma \mu-b \gamma y & -\gamma-\mu-b y & b y \\ 0 & 0 & 0\end{array}\right), \tilde{C}=\left(\begin{array}{ccc}1 & 0 & 0\end{array}\right)$, and $\tilde{K}(y)=\left(\begin{array}{c}k_{1}(y) \\ k_{2}(y) \\ \Lambda(y)\end{array}\right)$.

For any fixed $y>0$, it is not difficult to show that the pair $(\tilde{A}(y), \tilde{C}(y))$ is observable. That is, it satisfies the Kalman observability rank condition (see Definition 2.3). Therefore, by the pole-shifting theorem (see Theorem 2.1), it is possible to find a $y$-dependent gain $\tilde{K}(y)$ such that $\operatorname{sp}(M(y))=$ $\{-\lambda-2,-\lambda-1,-\lambda\}$, where $\operatorname{sp}(M(y))$ denotes the spectrum of $M(y)$ and $\lambda$ is any positive real number. This gain $\tilde{K}(y)$ is given by the relations (13) and can be computed using for instance Ackermann's formula (see [6] page 382):

$$
\tilde{K}(y)=\left(\tilde{A}(y)+\lambda I_{3}\right) \cdot\left(\tilde{A}(y)+(\lambda+1) I_{3}\right) \cdot\left(\tilde{A}(y)+(\lambda+2) I_{3}\right) \cdot \mathcal{O}^{-1}(y) \cdot\left(\begin{array}{l}
0 \\
0 \\
1
\end{array}\right)
$$

where $I_{3}$ is the $3 \times 3$ identity matrix and $\mathcal{O}(y)$ is the observability matrix associated to the pair $(\tilde{A}(y), \tilde{C}(y))$ and it is given by $\mathcal{O}(y)=\left(\begin{array}{c}\tilde{C}(y) \\ \tilde{C}(y) \tilde{A}(y) \\ \tilde{C}(y) \tilde{A}(y)^{2}\end{array}\right)$.

The spectrum of the matrix $M(y(t))$ is fixed, and lies entirely in the left hand side of the complex plane as the eigenvalues of $M(y(t))$ are $-\lambda-2,-\lambda-1$ and $-\lambda$, with $\lambda>0$. We must emphasize that, this, of course, is not sufficient to conclude that the solutions of $\dot{e}=M(y(t)) e$ converge to zero at an exponential rate since System (15) is a non autonomous system. Now, we will show that System (15) can be written in the form of $\dot{u}=(D+R(t)) u$, where $D$ is a constant matrix. Under some conditions on $R(t)$, the behavior of the solutions for these types of non autonomous systems could be obtained using Theorem 3.3 [20], for example.

Let $D=\operatorname{diag}(-\lambda,-(\lambda+1),-(\lambda+2))$ and $P(t)$ the invertible time-dependent matrix satisfying

$$
D=P^{-1}(t) M(y(t)) P(t)
$$

A straightforward computation of the eigenvectors of $M(y(t))$, associated with the corresponding eigen- 
values leads to determine $P(t)$ and thus $P^{-1}(t)$. We successively obtain

$$
P(t)=-b y(t)\left(\begin{array}{ccc}
-\frac{1}{(\lambda+1)(\lambda+2)} & -\frac{1}{\lambda(\lambda+2)} & -\frac{1}{\lambda(\lambda+1)} \\
\frac{\gamma+\mu-2 \lambda-3+b y(t)}{(\lambda+1)(\lambda+2)} & \frac{\gamma+\mu-2 \lambda-2+b y(t)}{\lambda(\lambda+2)} & \frac{\gamma+\mu-2 \lambda-1+b y(t)}{\lambda(\lambda+1)} \\
-\frac{1}{b y(t)} & -\frac{1}{b y(t)} & -\frac{1}{b y(t)}
\end{array}\right),
$$

and

$$
P^{-1}(t)=\left(\begin{array}{ccc}
-\frac{\lambda(\lambda+1)(\lambda+2)(\gamma+\mu-\lambda+b y(t))}{2 b y(t)} & -\frac{\lambda(\lambda+1)(\lambda+2)}{2 b y(t)} & \frac{(\lambda+1)(\lambda+2)}{2} \\
\frac{\lambda(\lambda+1)(\lambda+2)(\gamma+\mu-\lambda-1+b y(t))}{b y(t)} & \frac{\lambda(\lambda+1)(\lambda+2)}{b y(t)} & -\lambda(\lambda+2) \\
-\frac{\lambda(\lambda+1)(\lambda+2)(\gamma+\mu-\lambda-2+b y(t))}{2 b y(t)} & -\frac{\lambda(\lambda+1)(\lambda+2)}{2 b y(t)} & \frac{\lambda(\lambda+1)}{2}
\end{array}\right) .
$$

We perform the change of variable $u=P(t)^{-1} e$, and the new variable $u(t)$ satisfies the time-varing linear equation

$$
\dot{u}=(D+R(t)) u,
$$

where $R(t)=\frac{d P^{-1}(t)}{d t} P(t)$. A simple computation gives

$$
R(t)=\frac{y^{\prime}(t)}{y(t)}\left(\begin{array}{ccc}
\frac{1}{2} \lambda(\lambda-b y(t)+3) & \frac{1}{2}(\lambda+1)(\lambda-b y(t)+2) & \frac{1}{2}(\lambda+2)(\lambda-b y(t)+1) \\
-\lambda(\lambda-b y(t)+2) & -(\lambda+1)(\lambda-b y(t)+1) & -(\lambda+2)(\lambda-b y(t)) \\
\frac{1}{2} \lambda(\lambda-b y(t)+1) & \frac{1}{2}(\lambda+1)(\lambda-b y(t)) & \frac{1}{2}(\lambda+2)(\lambda-b y(t)-1)
\end{array}\right) .
$$

Therefore, it follows that

$$
\|R(t)\|_{\infty}=\left|\frac{y^{\prime}(t)}{y(t)}\right||(3(\lambda+1)(\lambda-b y(t))+3 \lambda+1)| .
$$

The solutions of of System (6) evolve in the compact set $\Omega$ defined in (7), so $y(t)(=w(t))$ is a positive and bounded function of $t$. We choose $\lambda$ large enough in such a way that $\lambda-b y(t) \geq 0$. Thus,

$$
\begin{gathered}
\|R(t)\|_{\infty}=\left|\frac{y^{\prime}(t)}{y(t)}\right|(3(\lambda+1)(\lambda-b y(t))+3 \lambda+1)=\left|\frac{y^{\prime}(t)}{y(t)}\right|\left(3 \lambda^{2}+6 \lambda+1-3 b(\lambda+1) y(t)\right) . \\
\|R(t)\|_{\infty}=\left(3 \lambda^{2}+6 \lambda+1\right)\left|\frac{y^{\prime}(t)}{y(t)}\right|-3 b(\lambda+1)\left|y^{\prime}(t)\right| .
\end{gathered}
$$

Since System (6) is a planar cooperative system there exists $T \geq 0$ such that $y(t)$ is nonincreasing or nondecreasing on $t \geq T$ (see [42], Chapter 3, Theorem 2.2, page 43, [28], Theorem 3.21, or [29], Proposition 2.5). This implies that $y^{\prime}(t)$ changes sign at most once (for $t=T$ ). Using Chasles' identity, we have $\int_{0}^{\infty}\|R(t)\|_{\infty} d t=\int_{0}^{T}\|R(t)\|_{\infty} d t+\int_{T}^{\infty}\|R(t)\|_{\infty} d t$, and 


$$
\int_{T}^{\infty}\|R(t)\|_{\infty} d t= \pm \int_{T}^{\infty} \frac{y^{\prime}(t)}{y(t)}\left(3 \lambda^{2}+6 \lambda+1-3 b(\lambda+1) y(t)\right) d t
$$

The sign in (17) is positive when $y^{\prime}(t) \geq 0$ and negative otherwise. Thus, we obtain, using the fact that $y(t)$ tends to $\bar{y}=\frac{a}{\gamma}\left(1-\frac{\gamma \mu}{a b}\right)$ as $t$ tends to $+\infty$

$$
\begin{aligned}
\int_{T}^{\infty}\|R(t)\|_{\infty} d t & = \pm\left[\left(3 \lambda^{2}+6 \lambda+1\right) \int_{T}^{\infty} \frac{y^{\prime}(t)}{y(t)} d t-3 b(\lambda+1) \int_{T}^{\infty} y^{\prime}(t) d t\right] \\
& = \pm\left[\left(3 \lambda^{2}+6 \lambda+1\right) \log \left(\frac{\bar{y}}{y(T)}\right)-3 b(\lambda+1)(\bar{y}-y(T))\right] \\
& <\infty
\end{aligned}
$$

Hence, $\int_{0}^{\infty}\|R(t)\|_{\infty} d t<\infty$, and so we can apply [20] (Chap. IV, Theorem 2): the solutions of system (16) satisfy for $t \rightarrow \infty$

$$
u_{i}(t) \sim e^{-\lambda_{i} t}, \text { for } i=1,2,3 .
$$

Going back to the error $e(t)=P(t) u(t)$, we obtain, for $t$ sufficiently large, $|e(t)| \leq \chi(y(t)) e^{-\lambda t}|e(0)|$ with $\chi$ being a positive continuous function. Since $y(t)$ is a continuous bounded function, we deduce that $|e(t)| \leq C_{2} e^{-\lambda t}|e(0)|$, for some positive constant $C_{2}$. This ends the proof.

The observer in Theorem 3.2 gives an estimation $\hat{z}$ of $z$ and, $\hat{a}$ of $a$. Hence, using the linear and invertible transformation $z=T x$, we obtain an estimation of $x$. Indeed, in the original coordinates $\hat{x}=(\hat{w}, \hat{s})$, the adaptive observer $(14)$ is given by:

$$
\left\{\begin{array}{l}
\dot{\hat{x}}(t)=\left(\begin{array}{cc}
-\gamma & \hat{a}(t) \\
b & -\mu-b y(t)
\end{array}\right) \hat{x}(t)-\left[\left(\begin{array}{cc}
1 & 0 \\
\frac{\gamma}{\hat{a}(t)} & \frac{1}{\hat{a}(t)}
\end{array}\right) K(y(t))-\frac{\hat{s}(t)}{\hat{a}(t)}\left(\begin{array}{c}
0 \\
\Lambda(y(t))
\end{array}\right)\right]\left(C_{0} \hat{x}(t)-y(t)\right) \\
\dot{\hat{a}}(t)=-\Lambda(y(t))\left(C_{0} \hat{x}(t)-y(t)\right)
\end{array}\right.
$$

It is worthwhile noting that the estimators (14) and (18) are completely independent of $a$.

Figure 3 provides the dynamics of $z_{2}(t)$ (red), solution the shifted system (12) and that of its estimate $\hat{z}_{2}(t)$ (dashed blue), obtained using the corresponding observer (14). The dynamics of the original variable $s(t)$ and its estimate $\hat{s}(t)$ are represented in Figure 4. The estimate $\hat{s}(t)$ is obtained using the adaptive observer, in original coordinates. That is, System (18). Also, as expected from Theorem 3.2, $\hat{z}_{2}(t)$ (Figure 3 ) and $\hat{s}(t)$ (Figure 4 ) converge exponentially to $z_{2}(t)$ and $s(t)$ respectively. Moreover, the efficiency of the designed observer is showcased in Figure 4, as it can be noticed that the estimated state $\hat{s}(t)$ converges toward the state $s(t)$ much earlier than they both reach the equilibrium value (dashed green line). 


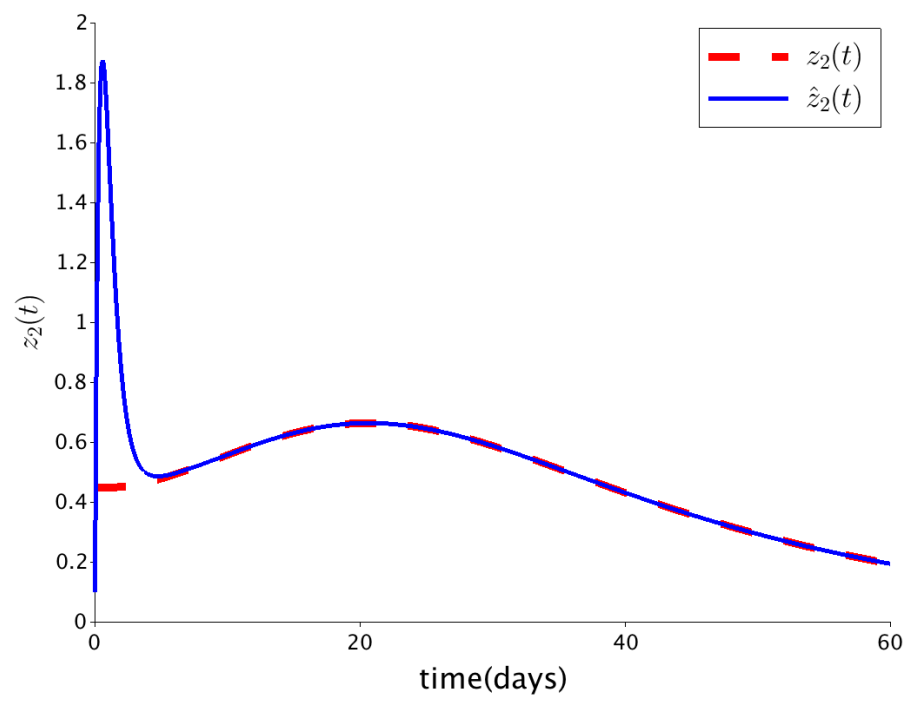

Figure 3: Dynamics of the state variable $z_{2}(t)$ (dashed red) given by system (12) and its corresponding estimate $\hat{z}_{2}(t)$ (blue) delivered by the observer (14). The initial conditions are such that $\left(z_{1}(0), z_{2}(0)\right)=$ $(3,0.3)$ and $\left(\hat{z}_{1}(0), \hat{z}_{2}(0)\right)=(1,0.1)$.

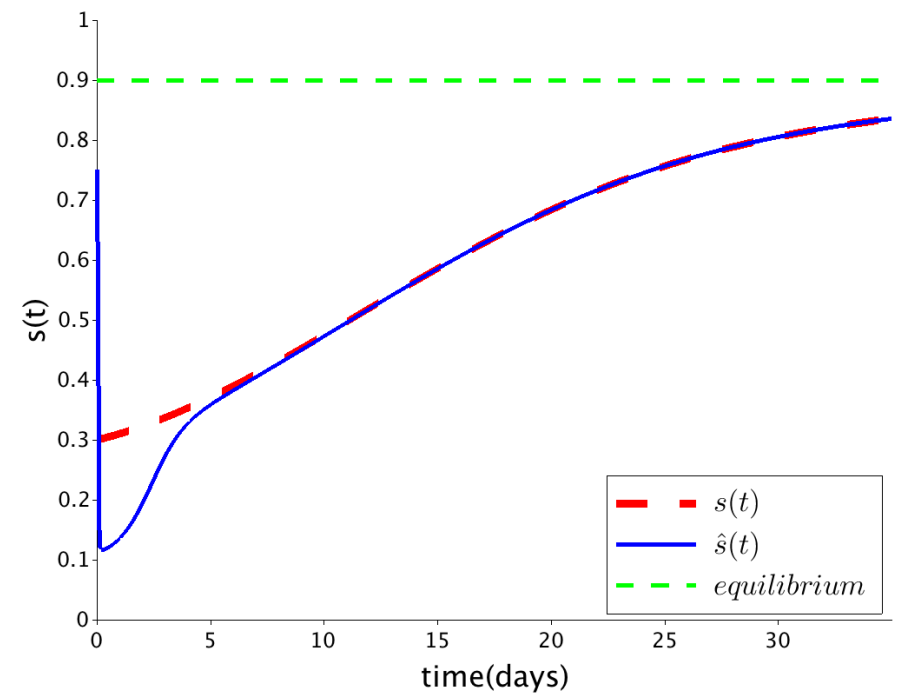

Figure 4: Dynamics of proportion of infected snails $s(t)$ (dashed red) and its estimate $\hat{s}(t)$ (blue) delivered by the observer (18). The estimation $\hat{s}(t)$, of $s(t)$ is obtained without using the unknown parameter $a$. The initial conditions are such that $(w(0), s(0))=(3,0.3)$ and $\left(\hat{z}_{1}(0), \hat{z}_{2}(0)\right)=(1,0.1)$. The green dashed line corresponds to the equilibrium value of $s(t)$, using Model (6). 


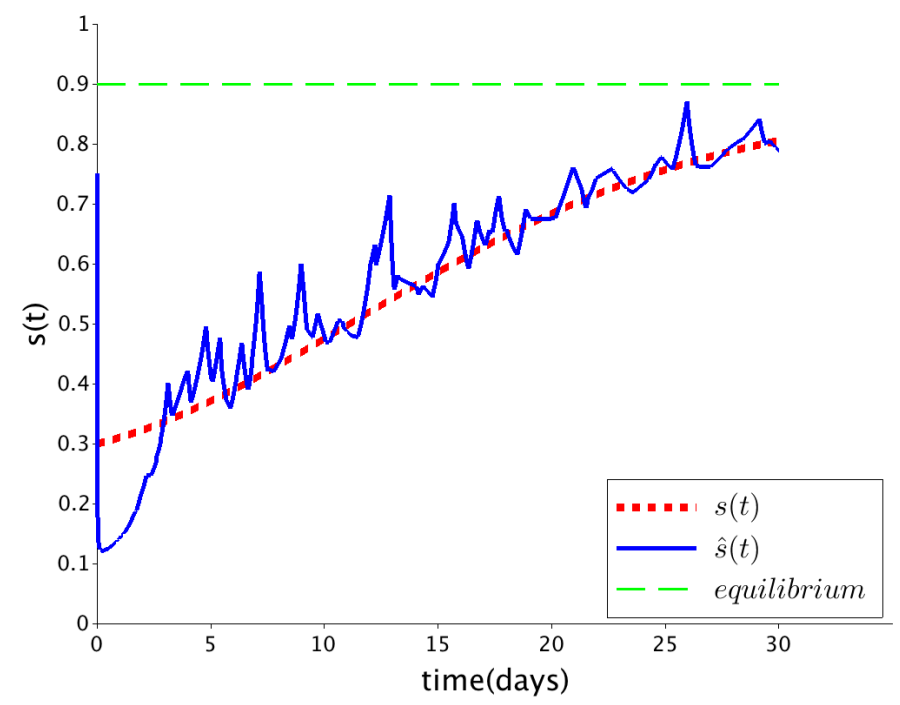

Figure 5: Dynamics of proportion of infected snails $s(t)$ (dotted red curve) and its estimate $\hat{s}(t)$ (blue) when the measurements $y(t)$ are corrupted by noise. The estimation $\hat{s}(t)$, of $s(t)$, is obtained without using the unknown parameter $a$, using the adaptive observer (18).

Remark 3.2. The observer (9) and the adaptive observer (18) both estimate the proportion of infected snails $s(t)$, and for our model and baseline parameter values, numerical simulations indicate that the estimations given by both observers converge to the state variables of Model (8) with approximately the same time period of around 5 days (see Figure 1 and Figure 4). However, the adaptive observer (18) allows to estimate not only the unmeasured state variables but also the unknown snail-man transmission parameter a. Moreover, numerical simulations suggest that the adaptive observer (18) is less sensitive to measurement noise as it can be seen by comparing Figure 5 and Figure 2.

Remark 3.3. The state and parameters estimation problem for the MacDonald's model (6) has been addressed in [45] using high-gain observer (we refer to [25] for a comprehensive introduction of a highgain observer). However, the use of high-gain observer requires the construction of a prolongation of the vector field that has to be globally Lipschitz not only on the set $\Omega$ but on the whole $\mathbb{R}^{2}$. It is also necessary to extend the diffeomorphism (defining the coordinates change) to the whole $\mathbb{R}^{2}$ and this extended diffeomorphism must also be globally Lipschitz [25]. The explicit construction of these extensions is not an easy task. The high-gain observer may fail to converge if these Lipschitz extensions are not used as it has been shown in [27].

In Theorem 3.1, we provided an estimation of the proportion of infected snails $(s(t))$ when all parameters are supposedly known. And, in Theorem 3.2, we designed an adaptive observer to estimate simultaneously both the state variable $s(t)$ and a key unknown parameter - the snail-to-human transmission rate. However, these estimations correspond to our particular set of data, and thus may not be used in the context of the actual disease control. Indeed, these estimations are based on Macdonald's model, a crude representation of the dynamics of Schistosomiasis. To address parts of the coarseness 


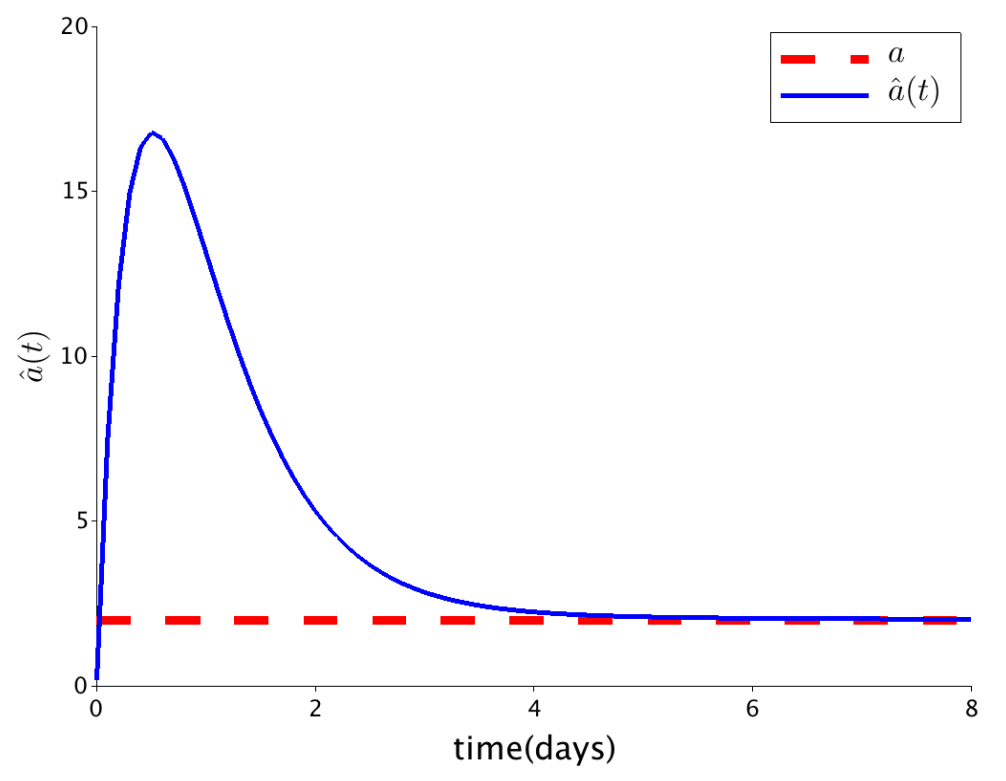

Figure 6: Comparison between the estimation of the unknown parameter $a$, as limit of the solution $\hat{a}(t)$ (blue) of the estimator (14) or (18) and the value of $a$, taking in the simulations of Model (6). It can be observed that the estimation convergence is quite fast.

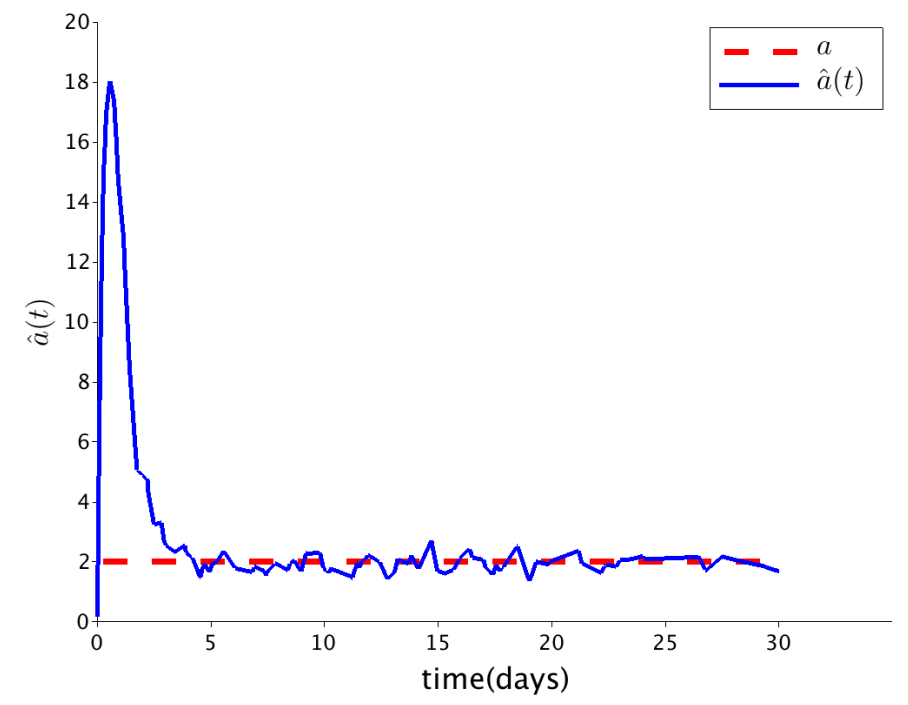

Figure 7: Comparison between the estimation of the unknown parameter $a$, as limit of the solution $\hat{a}(t)$ (blue curve) of the estimator (14) when the measurements $y(t)$ are corrupted by noise and the value of $a$, taking in the simulations of Model (6). It can be observed that the estimation convergence is quite fast. 
of Macdonald's model, we provide in the following subsection an estimation when the infection process is represented by a function $f(w, s)$, instead of an exponential distribution.

\subsection{State Estimate when the snail-host infection process is not known}

In estimating the proportion of infected snails, we used MacDonald's model (6), for which the snailhuman infection term is linear, namely " $a s$ ". Here, we propose a tool that allows to estimate the proportion of infected snails when the infection process is captured by any function $f(w, s)$ whose analytic expression is not necessarily known. Indeed, in this case, the modified MacDonald's model could be written as

$$
\left\{\begin{array}{l}
\dot{w}=-\gamma w+f(w, s) \\
\dot{s}=b(1-s) w-\mu s \\
y=w
\end{array}\right.
$$

or equivalently,

$$
\left\{\begin{array}{l}
\dot{x}=G x+E f(x)+g(x) \\
y=C x
\end{array}\right.
$$

where

$x=(w, s)^{T}, G=\left(\begin{array}{cc}-\gamma & 0 \\ b & -\mu\end{array}\right), E=\left(\begin{array}{l}1 \\ 0\end{array}\right), C=(1,0)$, and $g(w, s)=\left(\begin{array}{c}0 \\ -b s w\end{array}\right)=\left(\begin{array}{c}0 \\ -b y s\end{array}\right)$

Theorem 3.4. Let $z(t)=\left(z_{1}(t), z_{2}(t)\right)^{T}, \bar{G}=\left(I_{2}-E C\right) G, K=\left(k_{1}, k_{2}\right)$, with $k_{1}>0, k_{2}=b>0$ and $\hat{g}(t)=\left(0,-b y z_{2}\right)^{T}$. The following system

$$
\left\{\begin{array}{l}
\dot{z}=(\bar{G}-K C) z+(K+(\bar{G}-K C) E) y+\hat{g} \\
\hat{x}=z+E y
\end{array}\right.
$$

is an exponential observer for system (19), that is, $\hat{x}(t)$ converges exponentially towards $x(t)$.

Proof.

The observer construction is inspired from [30]. Let us decouple the System (19) from the unknown input $f(w, s)=f(x)$. Indeed, from Equation (20b), it follows that

$$
\begin{aligned}
\dot{y} & =C \dot{x} \\
& =C(G x+E f(x)+g(x)) \\
& =C G x+C E f(x)+C g(x) .
\end{aligned}
$$

Since $C E=1$, we deduce that $f(x)=\dot{y}-C G x-C g(x)$. Thus, Equation (20a) will be decoupled from the unknown input $f(x)$. Indeed, we obtain:

$$
\dot{x}=\bar{G} x+E \dot{y}+\left(I_{2}-E C\right) g(x) .
$$


Now it suffices to investigate the dynamics of the error $e(t):=\hat{x}(t)-x(t)$ between Systems $(20)$ and (21). We have:

$$
\begin{aligned}
\dot{e} & =\dot{\hat{x}}-\dot{x} \\
& =(\bar{G}-K C) e+\hat{g}-\left(I_{2}-E C\right) g(x) \\
& =(\bar{G}-K C) e-b y\left(\begin{array}{ll}
0 & 0 \\
0 & 1
\end{array}\right) e \\
& =\left(\begin{array}{cc}
-k_{1} & 0 \\
b-k_{2} & -\mu-b y
\end{array}\right) e .
\end{aligned}
$$

We choose $k_{2}=b$, we then have $\frac{d}{d t}\left(\|e(t)\|_{2}^{2}\right)=2\left(-k_{1} e_{1}^{2}-(\mu+b y(t)) e_{2}^{2}\right)$. Since $y(t) \geq 0$, it follows that $\frac{d}{d t}\left(\|e(t)\|_{2}^{2}\right) \leq-2\left(k_{1} e_{1}^{2}+\mu e_{2}^{2}\right) \leq-2 \min \left(k_{1}, \mu\right)\|e(t)\|_{2}^{2}$. Hence, $\|e(t)\|_{2}^{2} \leq e^{-2 \min \left(k_{1}, \mu\right) t}\|e(0)\|_{2}^{2}$ which shows that the estimation error $e(t)$ converges exponentially towards zero. This ends the proof.

Remark 3.4. System (21) is called observer with unknown input [15, 21, 30, 31].

\section{Observer design for a patchy Schistosomiasis model}

In this section, we propose to design an observer for Schistosomiasis model that accounts for individuals and ponds heterogeneity. Indeed, we revisit a dynamical model proposed in [40] for which individuals are structured into $l$ patches or groups that interact with the snails that are dispatched in $m$ freshwater contact sites or pond locations. Using the same setup as the homogeneous case and by denoting $w=\left(w_{1}, w_{2}, \ldots, w_{l}\right)$ the vector corresponding to the number of female schistosomes (worms) per host (for $l$ patches or groups) and $s=\left(s_{1}, s_{2}, \ldots, s_{m}\right)$ the vector corresponding to the proportion of infected snails (for $m$ freshwater sites or pond locations). Hence, following [40], the dynamics of Schistosomiasis that results from the interactions of $m$ groups of individuals into $l$ freshwater sources is given by:

$$
\left\{\begin{array}{l}
\dot{w}=-\operatorname{diag}(\gamma) w+A s \\
\dot{s}=\operatorname{diag}(\mathbb{1}-s) B w-\operatorname{diag}(\mu) s
\end{array}\right.
$$

where $A=\left(a_{i j}\right) \in M_{l, m}(\mathbb{R})$ is a nonnegative $l \times m$ matrix, which captures the snail to man transmission, and $B=\left(b_{i j}\right) \in M_{m, l}(\mathbb{R})$ a nonnegative $m \times l$ matrix, which captures the man to snail transmission. More precisely, $a_{i j}$ is the transmission from snails in Pond $j$ to humans of Patch $i$ and $b_{i j}$ is the transmission from humans of Patch $i$ to snails of Pond $j$. As for the homogeneous case, $\gamma \in \mathbb{R}_{+}^{l}$ is the vector of death rates of female schistosome in the human host populations, $\mu \in \mathbb{R}_{+}^{m}$ is the vector of death rates of snails in the water site. By denoting $\mathbb{1}$, the vector $(1, \cdots, 1)^{T}$ of $\mathbb{R}^{m}$, it could be shown easily (see [40] for instance) that the set

$$
\mathcal{D}=\left\{(w, s) \in \mathbb{R}_{+}^{l+m} \mid 0 \leq w \leq \operatorname{diag}(\gamma)^{-1} A \mathbb{1} ; 0 \leq s \leq \mathbb{1}\right\}
$$


is an absorbing and positively invariant set for system (23), thereby making the solutions of (23) biologically grounded. A detailed formulation of Model (23) and its dynamics can be found in [9, 38, 40] for example.

We assume that the measurable output is the vector $w$. That is, we measure the worms in humans host in each group. Noting that $\operatorname{diag}(\mathbb{1}-s) B w-\operatorname{diag}(\mu) s=B w-\operatorname{diag}(B w) s-\operatorname{diag}(\mu) s$, and since the measurable output is $y=w$, Model (23) along with the measured data could be written as

$$
\left\{\begin{array}{l}
\dot{x}=M x+U(y) x \\
y=C x
\end{array}\right.
$$

where

$$
x=(w, s)^{T}, \quad M=\left(\begin{array}{cc}
-\operatorname{diag}(\gamma) & A \\
B & -\operatorname{diag}(\mu)
\end{array}\right), U(y)=\left(\begin{array}{cc}
0 & 0 \\
0 & -\operatorname{diag}(B y)
\end{array}\right)
$$

and

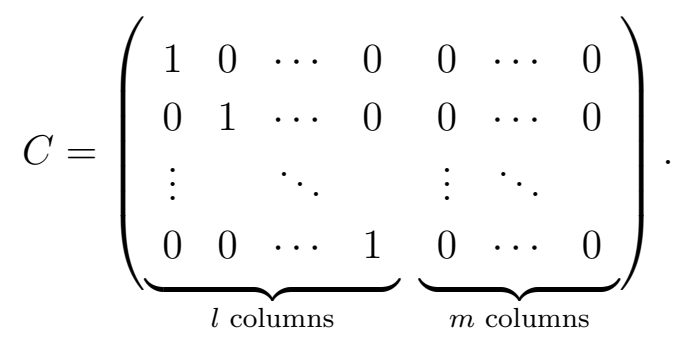

The following theorem gives an exponential observer for System (24).

\section{Theorem 4.1.}

An exponential state estimator for System (24) is given by

$$
\dot{\hat{x}}=M \hat{x}+U(y) \hat{x}-K(C \hat{x}-y)
$$

where $K=\left(\begin{array}{c}K_{1} \\ K_{2}\end{array}\right)$ with $K_{2}=A^{T}+B$, and $K_{1}$ any $l \times l$ square matrix whose components are all positive.

Proof. Let $e=\hat{x}-x$, the error between System (25) and System (24). The evolution of the error equation is $\dot{e}=(M+U(y)-K C) e$. Considering the function $V=\frac{1}{2} e^{T} e$, we obtain

$$
\dot{V}=-e_{1}^{T}\left(K_{1}^{T}+\operatorname{diag}(\gamma)\right) e_{1}-e_{2}^{T}(\operatorname{diag}(\mu)+\operatorname{diag}(B y)) e_{2}+e_{2}^{T}\left(A^{T}+B-K_{2}\right) e_{1}
$$

Thus, it is possible to find $K_{1}$ and $K_{2}$ that make $\dot{V}$ is negative definite. A simple choice is $K_{2}=A^{T}+B$ and $K_{1}>0$.

The observer obtained in Theorem 4.1 enables the estimation of the proportion of infected snails in each freshwater site. This is particularly useful in providing a tool to better control Schistosomiasis, such as biological control of snails [43] or in allocating resources (such as the use of chemical molluscicides [35]) at appropriate snails' freshwater habitats according to their infected snails importance. 


\section{Conclusion and discussions}

In this paper, we investigated tools and methods to estimate state variables and parameters of a class of schistosomiasis models using elements of control theory. Particularly, we address two fundamental problems in modeling and control of schistosomiasis transmission. Namely, we propose a method to estimate the proportion of infected snails in a given snail habitat and the snail-host transmission parameter. We revisited the classical MacDonald's model [4, 8, 9] for schistosomiasis and used the fact that a key state variable could be measured, namely the worms burden in humans. Our method consists of using elements of system theory in designing an auxiliary dynamical system, called observer, that converges exponentially to the original model.

First, we estimated the proportion of infected snails, assuming that all parameters are known (Theorem 3.1) and when the snail-host transmission parameter is unknown (Theorem 3.2) in a homogeneous system. Moreover, using the latter observer, we estimated the unknown parameter. We then compared the asymptotic value of the obtained parameter with that a constant valued used in the dynamics of Schistosomiasis model (Figure 6).

Second, we considered a heterogeneous schistosomiasis model with an arbitrary number of age-group in the structure of human hosts and where the snails are distributed into $m$ water sources, considered in $[9,38,40])$. We derived a method to estimate the proportion of infected snails in each snail habitat (Theorem 4.1). This result, we hope, could help in better designing effective public health policies, particularly in allocating spare resources where they are needed the most, with respect to host groups or snail habitats.

In both the homogeneous and heterogeneous cases, we estimated the snail-human transmission rate. However, the human-snail transmission $b$ is also unknown. This parameter become the WAIFW (Who Acquires Infection From Whom [5]) matrix $B$ for the heterogeneous case. This is very challenging as the cross term is nonlinear and is the subject of an ongoing investigation. In this paper, the observers are designed with the assumption that the measurable output is a function continuous in time. However, in real life, one can hope at best to have a limited number of samples of measurements at discrete times. It would be interesting to design observer for epidemic models - that are continuous in time for which the measurable output is discrete in time.

\section{Acknowledgements}

We are grateful to two anonymous referees and the handling editor for valuable comments and suggestions that led to an improvement of this paper. 


\section{References}

[1] A. Abdelhedi, D. Boutat, L. Sbita, and R. Tami, Extended observer to estimate the spreading of contagious disease, in 2014 European Control Conference (ECC), June 2014, pp. 1843-1848.

[2] A. Abdelhedi, D. Boutat, L. Sbita, R. TAmi, And D.-Y. Liu, Observer design for a class of nonlinear piecewise systems. Application to an epidemic model with treatment, Math. Biosci., 271 (2016), pp. 128-135.

[3] J. Alvarez-Ramirez, M. Meraz, and J. X. Velasco-Hernandez, Feedback control of the chemotherapy of HIV, International Journal of Bifurcation and Chaos, 10 (2000), pp. 2207-2219.

[4] R. M. Anderson And R. M. May, Helminth infections of humans: mathematical models, population dynamics, and control, Advances in parasitology, 24 (1985), pp. 1-101.

[5] R. M. Anderson And R. M. May, Infectious Diseases of Humans. Dynamics and Control, Oxford science publications, 1991.

[6] P. J. Antsaklis And A. N. Michel State Feedback and State Observers, pages 351-410. Birkhäuser Boston, Boston, MA, 2007.

[7] M. S. Aronna And P.-A. Bliman, Interval observer for uncertain time-varying SIR-SI model of vector-borne disease, in Proc. of European Control Conference, 2018.

[8] A. Barbour, Modeling the transmission of schistosomiasis: an introductory view, The American journal of tropical medicine and hygiene, 55 (1996), pp. 135-143.

[9] A. D. Barbour, Macdonald's model and the transmission of bilharzia, Transactions of the Royal Society of Tropical Medicine and Hygiene, 72 (1978), pp. 6-15.

[10] M.-G. Basáñez, J. S. McCarthy, M. D. French, G.-J. Yang, M. Walker, M. Gambhir, R. K. Prichard, And T. S. Churcher, A research agenda for helminth diseases of humans: modelling for control and elimination, PLoS neglected tropical diseases, 6 (2012), p. e1548.

[11] G. BesanÇOn, ed., Nonlinear observers and applications. Papers based on the presentations at the 28th Grenoble international summer school on control, Grenoble, France, September 200\%., Lecture Notes in Control and Information Sciences 363. Berlin: Springer. xiii, 224 p. , 2007.

[12] G. Besancon, Q. Zhang, and H. Hammouri. High gain observer based state and parameter estimation in nonlinear systems. In NOLCOS 2004, 2004.

[13] J. Bethony, J. T. Williams, H. Kloos, J. Blangero, L. Alves-Fraga, G. Buck, A. Michalek, S. Williams-Blangero, P. T. LoVerde, R. Corréa-Oliveira, and A. GAzZINELli, Exposure to schistosoma mansoni infection in a rural area in brazil. ii: household risk factors, Tropical Medicine \& International Health, 6 (2001), pp. 136-145. 
[14] D. Bichara, Étude de modèles épidémiologiques: Stabilité, observation et estimation de paramètres, PhD thesis, Université de Lorraine, 2013.

[15] D. Bichara, N. CozIc, And A. IGgidr, On the estimation of sequestered infected erythrocytes in Plasmodium falciparum malaria patients, Math. Biosci. Eng., 11 (2014), pp. 741-759.

[16] P.-A. Bliman and B. D’Avila Barros, Interval observers for SIR epidemic models subject to uncertain seasonality, in Positive systems, vol. 471 of Lect. Notes Control Inf. Sci., Springer, Cham, 2017, pp. 31-39.

[17] P.-A. Bliman, D. Efimov, and R. Ushirobira, A class of nonlinear adaptive observers for sir epidemic model, in Proc. of European Control Conference, 2018.

[18] Centers for Disease Control and Prevention, Neglected tropical diseases, Centers for Disease Control and Prevention, https://www.cdc.gov/globalhealth/ntd/fastfacts.html, (2017).

[19] D. Colley And W. Secor, Immunology of human schistosomiasis, Parasite immunology, 36 (2014), pp. 347-357.

[20] W. A. Coppel, Stability and asymptotic behavior of differential equations, D. C. Heath and Co., Boston, Mass., 1965.

[21] M. Darouach, M. Zasadzinski, and S. J. Xu, Full-order observers for linear systems with unknown inputs, IEEE transactions on automatic control, 39 (1994), pp. 606-609.

[22] K. Degue, D. Efimov, And A. IGgidR, Interval estimation of sequestered infected erythrocytes in malaria patients, 2016 European Control Conference, ECC 2016, (2017), pp. 1141-1145.

[23] M. Diaby, A. IGGidR, AND M. Sy, Observer design for a schistosomiasis model, Mathematical Biosciences, 269 (2015), pp. $17-29$.

[24] Z. Feng, A. Eppert, F. A. Milner, And D. Minchella, Estimation of parameters governing the transmission dynamics of schistosomes, Applied Mathematics Letters, 17 (2004), pp. 11051112.

[25] J. P. Gauthier, H. Hammouri, and S. Othman, A simple observer for nonlinear systems applications to bioreactors, IEEE Transactions on automatic control, 37 (1992), pp. 875-880.

[26] D. J. Gray, D. P. Mcmanus, Y. Li, G. M. Williams, R. Bergquist, And A. G. Ross, Schistosomiasis elimination: lessons from the past guide the future, Gray, Darren J and McManus, Donald P and Li, Yuesheng and Williams, Gail M and Bergquist, Robert and Ross, Allen G, 10 (2010), pp. 733-736.

[27] A. Guiro, A. IgGidr, D. NGom, And H. TourÉ, On the stock estimation for some fishery systems, Reviews in fish biology and fisheries, 19 (2009), pp. 313-327. 
[28] M. Hirsch And H. L. Smith, Handbook of Differential Equations 2, Elsevier, 2005, ch. Monotone Dynamical Systems.

[29] M. W. Hirsch, Systems of differential equations which are competitive or cooperative. I: Limit sets., SIAM J. Math. Anal., 13 (1982), pp. 167-179.

[30] M. Hou And P. Muller, Design of observers for linear systems with unknown inputs, IEEE Transactions on automatic control, 37 (1992), pp. 871-875.

[31] M. Hou And P. Muller, Disturbance decoupled observer design: A unified viewpoint, IEEE Transactions on Automatic Control, 39 (1994), pp. 1338-1341.

[32] A. IGGidR, Encyclopedia of life support systems (eolss), in Mathematical Models, J. A. Filar, ed., UNESCO, Eolss Publishers, Oxford,UK, 2004, ch. Controllability, Observability and Stability of Mathematical Models.

[33] A. IGgidr And M. O. Souza, State estimators for some epidemiological systems, J Math Biol, (2018).

[34] M. T. Inobaya, R. M. Olveda, T. N. Chau, D. U. Olveda, and A. G. Ross, Prevention and control of schistosomiasis: a current perspective, Research and reports in tropical medicine, 2014 (2014), p. 65.

[35] C. H. King and D. Bertsch, Historical perspective: snail control to prevent schistosomiasis, PLoS neglected tropical diseases, 9 (2015), p. e0003657.

[36] D. Luenberger, An introduction to observers, IEEE Transactions on automatic control, 16 (1971), pp. 596-602.

[37] G. Macdonald, The dynamics of helminth infections, with special reference to schistosomes, Transactions of the Royal Society of Tropical Medicine and Hygiene, 59 (1965), pp. 489-506.

[38] L. Mari, M. Ciddio, R. Casagrandi, J. Perez-Saez, E. Bertuzzo, A. Rinaldo, S. H. Sokolow, G. A. De Leo, And M. Gatto, Heterogeneity in schistosomiasis transmission dynamics, Journal of Theoretical Biology, 432 (2017), pp. 87-99.

[39] K. S. Narendra and A. M. Annaswamy. Stable adaptive systems. Prentice-Hall, Inc., Upper Saddle River, NJ, USA, 1989.

[40] G. Riveau, G. Sallet, and L. Tendeng, A Transmission Model of Bilharzia A Mathematical Analysis of an Heterogeneous Model, Revue Africaine de la Recherche en Informatique et Mathématiques Appliquées, 14 (2011), pp. 1-13.

[41] R. Ross, The prevention of malaria, John Murray, 1911. 
[42] H. L. Smith, Monotone dynamical systems: an introduction to the theory of competitive and cooperative systems., Mathematical Surveys and Monographs. 41. Providence, RI: American Mathematical Society (AMS). x, 174 p. , 1995.

[43] S. J. Swartz, G. A. De Leo, C. L. Wood, and S. H. Sokolow, Infection with schistosome parasites in snails leads to increased predation by prawns: implications for human schistosomiasis control, Journal of Experimental Biology, 218 (2015), pp. 3962-3967.

[44] R. Tami, D. Boutat, G. Zheng, and F. Kratz, Parameters and states estimation for dengue epidemic model, in 2014 European Control Conference (ECC), June 2014, pp. 528-533.

[45] L. Tendeng, Etude de modèles de transmission de la Schistosomiase: Analyse mathématique, reconstruction des variables d'état et estimation des paramètres, $\mathrm{PhD}$ thesis, Université de Lorraine, 2013.

[46] G. Teschl. Ordinary Differential Equations and Dynamical Systems. Graduate studies in mathematics. American Mathematical Soc., 2012.

[47] J. Utzinger, R. Bergquist, X. Shu-Hua, B. H. Singer, and M. Tanner, Sustainable schistosomiasis control - the way forward, The Lancet, 362 (2003), pp. 1932-1934.

[48] H. J. Van Cleave And L. G. Lederer, Studies on the life cycle of the snail, viviparus contectoides, Journal of Morphology, 53 (1932).

[49] J. X. Velasco-Hernández, J. A. García, And D. Kirschner, Remarks on modeling hostviral dynamics and treatment., in Mathematical approaches for emerging and reemerging infectious diseases: An introduction. Proceedings of a tutorial Introduction to epidemiology and immunology, C. e. e. a. Castillo-Chavez, ed., Springer. IMA Vol. Math. Appl. 125, 2002, pp. 287-308.

[50] W. Wonham. Linear multivariable control: a geometric approach. 2nd ed. Springer, 1979.

[51] M. Woolhouse, On the application of mathematical models of schistosome transmission dynamics. i. natural transmission, Acta tropica, 49 (1991), pp. 241-270.

[52] _ On the application of mathematical models of schistosome transmission dynamics. ii. control, Acta Tropica, 50 (1992), pp. 189-204.

[53] M. Woolhouse, G. Hasibeder, And S. Chandiwana, On estimating the basic reproduction number for schistosoma haematobium, Tropical Medicine \& International Health, 1 (1996), pp. 456-463.

[54] World Health Organization, Schistosomiasis, World Health Organization, http://www. who.int/schistosomiasis/disease/en/, (2017). 